\title{
Steps that count! A feasibility study of a pedometer-based, health- promotion intervention in an employed, South African population
}

\author{
J D Pillay, ${ }^{1,2} \mathrm{PhD}$; T L Kolbe-Alexander, ${ }^{1} \mathrm{PhD}$; K I Proper, ${ }^{3} \mathrm{PhD}$; W van Mechelen, ${ }^{1,3} \mathrm{PhD} ; \mathrm{E} \mathrm{V} \mathrm{Lambert},{ }^{1} \mathrm{PhD}$ \\ ${ }^{1}$ UCT/MRC Research Unit for Exercise Science and Sports Medicine, Faculty of Health Sciences, University of Cape Town, South Africa \\ ${ }^{2}$ Department of Basic Medical Sciences, Faculty of Health Sciences, Durban University of Technology, South Africa \\ ${ }^{3}$ Department of Public and Occupational Health, EMGO Institute for Health and Care Research, VU University Medical Centre, Amsterdam, The Netherlands
}

Corresponding author: J D Pillay (pillayjd@dut.ac.za)

\begin{abstract}
Background. The emergence of the pedometer as a useful motivational aid for increasing physical activity (PA) has supported its use in PA interventions.

Objectives. To examine the feasibility of a 10-week pedometer-based intervention complemented by regular motivational messages, to increase ambulatory PA; and to determine the minimum sample size required for a randomised, controlled trial (RCT).

Methods. Participants, sourced by convenience sampling of employees from an academic institution, were randomly assigned to either an intervention group (IG) $(n=11)$ or control group (CG) $(n=11)$, following baseline health measurements and blinded pedometer wear (week 1). Participants in the IG subsequently wore an unblinded pedometer (10 weeks) to self-monitor daily steps. Individualised messages using pedometer data (IG) and general motivational messages (IG and CG) were provided bi-weekly. Blinded pedometer wear (IG and CG) and a feedback questionnaire (IG) were completed at week 12. Pedometer data were compared between the IG and CG at week 12.

Results. Participants' perceptions of the intervention supported the benefit of the pedometer as a useful motivational aid and a reminder to increase steps per day. Occupational sitting time and inability to incorporate PA into daily routine emerged as the main barrier to adherence. Steps per day increased more in the IG (mean \pm standard deviation (SD) 996 \pm 1 748) than in the CG (mean \pm SD 97 \pm 750 ). Modest improvements were noted in all clinical measures (IG).

Conclusion. Based on the improvement of 1000 steps/day (IG), a minimum of 85 participants in the IG and CG, respectively, is required for a future RCT ( $80 \%$ power; $p<0.05)$. We recommend a minimum of 150 participants in each group to account for loss to follow-up and to allow for subgroup analyses.
\end{abstract}

S Afr J SM 2014;26(1):15-19. DOI:10.7196/SAJSM.500

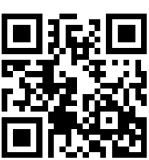

Physical inactivity is a global health concern ${ }^{[1]}$ with complex solutions, as behavioural change is often difficult to achieve and, more importantly, to sustain. ${ }^{[2]}$ Small behavioural changes may, however, be more feasible to achieve and maintain. ${ }^{[3]}$ Pedometers have been shown to offer a good solution for a low-cost, objective monitoring and behavioural modification tool and practical aid for physical activity (PA) interventions. ${ }^{[4-8]}$ Pedometers have therefore gained popularity for use in $\mathrm{PA}$ interventions in various settings ${ }^{[9]}$ to facilitate behavioural change.

Providing individualised feedback has been promoted as a useful adjunct to many health and well-being interventions, and has often been used as an additional support measure to pedometer-based interventions. ${ }^{[10]}$ A systematic review by Ogilvie et al ${ }^{[1]}$ in 2007 examined the effectiveness of interventions aimed at increasing the volume of walking. According to the review, the strongest evidence that exists supports interventions targeted at individuals motivated to change. ${ }^{[11]} \mathrm{A}$ further finding was that the interventions which involved strategies such as brief advice and the use of pedometers showed promising results, such as assistance with goal-setting, feedback on progress and strategies for overcoming barriers. ${ }^{[1]}$ There is, however, a large gap between the development of effective pedometer-based interventions and their feasibility for use in public health practice. ${ }^{[12,13]} \mathrm{A}$ primary limitation is the high cost and large time demands on both staff and participants. ${ }^{[13]}$ Using more cost-effective intervention strategies, such as pedometer-based approaches supplemented by email-based feedback, may overcome this limitation.

\section{Objectives}

The aims of this pilot study were therefore: (i) to evaluate the feasibility of a pedometer-based intervention complemented by individualised, email-based feedback promoting PA in an employed population; and (ii) to inform the development of, and calculate the recommended sample size for a pedometer-based intervention to be administered in the future as a randomised, controlled trial (RCT).

Methods

The pilot study was conducted at a tertiary academic institution in KwaZulu-Natal Province, South Africa, through convenience sampling. An advertisement was emailed to all staff members within the Faculty of Health Sciences inviting participation in the study.

Inclusion and exclusion criteria

All willing participants aged 21 - 49 years were eligible. Employees were excluded in the case of: pregnancy; diagnosis or treatment of cancer; 
any other physical/clinical condition that made PA difficult; contract workers whose employment with the company would end before the 12-week follow-up measurement; or non-compliance to a minimum of 3 days of blinded pedometer wear at baseline.

\section{Ethical considerations and pre-participation screening}

This study was conducted in accordance with the Declaration of Helsinki, Good Clinical Practice, as well as the ethical laws of South Africa. Ethical approval was obtained from the Human Research Ethics Committee of the Faculty of Health Sciences, University of Cape Town (reference 044/2009).

Following agreement to participate in the study, the physical activity readiness questionnaire (PAR-Q) $)^{[14]}$ was administered to participants to ensure that there were no health risks associated with participation in PA. Employees who agreed to study participation $(N=25)$ were asked to sign an informed consent form prior to participation, and were assured that participation was voluntary and that they could withdraw at any time without penalty. The participants were also assured that their employer would not have access to any of the information collected, and that all information would be maintained as strictly confidential.

\section{Measurements}

Body height $(\mathrm{cm})$ was measured using a height chart as the vertical distance from the floor to the vertex of the head. The participant stood barefoot with heels, buttocks and head in contact with the wall and arms at their side. Waist circumference $(\mathrm{cm})$ was measured using a tape measure around the skin of the waist. Body weight was measured using an electronic scale (Beurer PS 06), allowing only a single layer of clothing. The values were rounded to the nearest $100 \mathrm{~g}$. Body mass index (BMI) was computed as weight $(\mathrm{kg})$ divided by height $(\mathrm{m})$ squared. The Omron Body Composition Monitor (BF500) was used to measure percentage body fat, based on the principles of bioelectrical impedance. ${ }^{[15]}$ Blood pressure $(\mathrm{BP})$ was recorded $(\mathrm{mmHg})$ using a sphygmomanometer after the participant remained relaxed for $5 \mathrm{~min}$. Two readings were taken, approximately $5 \mathrm{~min}$ apart, and an average of the two readings was recorded. If the two were different from each other ( $>5 \mathrm{mmHg}$ ), a third reading was taken. The average of the two nearest readings was then used.

Following the 10-week intervention, both the IG and CG were required to complete follow-up measures, as in week 1. Participants in the IG were invited to complete a questionnaire reporting their perceptions of the intervention. A section was included on general comments and/or suggestions for improvement of the intervention. Both of these measures were used to inform the feasibility of the intervention.

\section{Baseline pedometer wear}

All participants were required to wear an Omron HJ 750 ITC pedometer attached to the hip area for 5 consecutive days. Participants were asked to wear the pedometer throughout the day and to follow their usual routine of daily activities and to remove the pedometer only when bathing, showering or swimming. The pedometer screen was covered to reduce the likelihood of participants observing their daily steps, which might have influenced habitual levels of PA and subsequently the accumulation of daily steps during the baseline measurement.
Participants were informed that their daily results would be made available to them at the end of the 5-day period.

\section{Allocation of participants to groups}

Following the baseline feedback on participants' PA levels from the data collected from the pedometers, participants were randomly allocated to an intervention group (IG) or a wait-listed control group (CG). By this random allocation, participants allocated to the CG would be offered the intervention following the study. The allocation of participants into IG and CG was achieved by random selection of participants from a composite participant list (Microsoft Excel).

IG participants were provided with an unblinded pedometer for the subsequent 10 weeks. They were shown how to upload and interpret their pedometer data, and were requested to provide the pedometer data to the researcher, via email, bi-weekly.

\section{Pedometer feedback}

Upon return of the pedometer at baseline, data regarding steps per day were electronically uploaded by the researcher according to the Omron Health Management Manager software protocol. ${ }^{[16]}$ This information was provided to participants. A unique feature of the pedometer is the ability to provide an hourly representation of steps per day. In addition to indicating total steps per day, the output can illustrate steps accumulated as being 'aerobic' or 'non-aerobic', according to the Omron classification that integrates both intensity and duration. The number of steps classified as aerobic (>60 steps/min; minimum duration $1 \mathrm{~min}$ ) and non-aerobic ( $<60 \mathrm{steps} / \mathrm{min}$ and/or duration $<1 \mathrm{~min}$ ) within the total steps per day record was therefore provided. Similarly, total time spent accumulating aerobic steps (in min/day; aerobic time) could be identified.

The PA recommendation of accumulating 30 min of moderate PA at least five times a week ${ }^{[1]}$ was reinforced to all participants (i.e. IG and CG). All participants were encouraged to improve their PA levels steadily (e.g. by $10 \%$ per week, until 30 min of moderate PA was achieved) during the subsequent 10 weeks. The phrase 'steps that count!' was adopted as a strategy for engaging people into accumulating intensity-based steps.

\section{Intervention}

Participants in the IG continued to wear the pedometer (unblinded) for the subsequent 10 weeks.

Following the electronic, bi-weekly receipt of pedometer data from IG participants, IG participants were provided with individualised, emailed feedback and a generalised information sheet on ways to increase PA. The individualised feedback included information on the average daily steps per day accumulated, the highest number of steps per day accumulated by the individual over the past 2 weeks, the number of days (if any) that aerobic steps per day were accumulated, and the volume thereof in the form of a personalised email. The general supportive/motivational messages included a key message (such as 'be active everyday' or 'walk tall'). A few strategies to increase PA (e.g. 'use the stairs instead of the lift/escalator'; 'walk fast enough so as to increase your breathing rate yet not feel out of breath') were also suggested. The purpose of the bi-weekly email was to provide a summary of daily steps accumulated and to suggest some strategies to 'add steps' to one's day. 
CG participants were similarly provided with a general motivational message (as in the IG) bi-weekly, but were not provided with a pedometer over the 10 weeks, and therefore no pedometer feedback.

\section{Follow-up measures}

The IG and CG were required to repeat the measurements that were conducted at baseline during the week immediately after the 10week intervention. Participants of the IG were invited to complete a questionnaire relating to their perceptions of the pilot intervention.

\section{Outcome measures}

Participants' perceptions of the intervention, in terms of the value, appeal, support and benefits of the intervention, were identified. This served as a primary outcome measure in determining the feasibility of the intervention. A section on general comments and suggestions for improvement of the intervention was included. This provided insights into strengths and areas for improvement so that a more effective intervention could subsequently be developed and applied. The secondary outcome measures (daily PA levels in terms of steps per day as well as biometric and clinical measures) were assessed at baseline (week 1) and follow-up (week 12) for both IG and CG. This allowed us to detect changes in daily ambulatory PA and biometric measures over time as a function of the intervention. Data were derived from the pedometer and expressed as steps per day. More importantly, information on the volume of sustained and moderate to vigorous intensity steps was assessed at baseline (week 1) and follow-up (week 12) for both the IG and CG. The change in steps per day at the end of the intervention directed us towards establishing an adequate sample size for a future RCT protocol.

\section{Statistical analyses}

Table 1 summarises general study group characteristics. Because of the pilot nature of this feasibility trial, we did not present statistical differences between groups for any of our measures. A descriptive analysis regarding barriers and facilitators of the intervention was performed on the information gathered from the questionnaire. From the questionnaire feedback, common emerging themes were identified in addition to facilitating factors and barriers to improving PA. Participants' perceptions on the value of the intervention were also identified so as to determine the feasibility of the intervention.

\section{Results}

Of the 25 participants who indicated an interest in study participation, 22 completed the baseline measures and pedometer wear and were randomly allocated to the CG or IG. Two CG participants, upon being allocated to the CG, declined further participation in the study and one CG participant did not complete the pedometer wear and followup measures at week 12. A total of 11 IG (two male; nine females) and eight CG (one male, seven females) participants completed follow-up measures and were included in the final analysis. The data indicated that of the 11 IG participants, three accumulated some aerobic steps at baseline. None of the participants in the CG accumulated aerobic steps.

It is noted that the IG was categorised into the 'obese' classification for BMI, while the CG was in the 'overweight' category for BMI. It was
Table 1. Descriptive baseline characteristics of participants $(N=19)$

\begin{tabular}{lll}
\hline Variable $^{*}$ & CG $(N=\mathbf{8})$ & IG $(N=11)$ \\
\hline Age (years) & $38.3 \pm 7.7$ & $37.6 \pm 8.6$ \\
Body height $(\mathrm{cm})$ & $168.9 \pm 5.0$ & $165.1 \pm 8.3$ \\
Body waist $(\mathrm{cm})$ & $84.6 \pm 9.9$ & $91.9 \pm 13.7$ \\
Body fat $(\%)$ & $36.0 \pm 9.5$ & $42.8 \pm 8.9$ \\
BMI $\left(\mathrm{kg} / \mathrm{m}^{2}\right)$ & $26.0 \pm 4.5$ & $31.1 \pm 6.6$ \\
Body weight $(\mathrm{kg})$ & $74.4 \pm 14.0$ & $84.4 \pm 17.4$ \\
SBP (mmHg) & $118 \pm 16$ & $118 \pm 13$ \\
DBP (mmHg) & $80 \pm 11$ & $85 \pm 14$ \\
Total steps per day (steps/day) & $4600 \pm 2041$ & $5370 \pm 1739$ \\
Aerobic steps (steps/day) & 0 & $331 \pm 646$ \\
Aerobic time (min) & 0 & $19.3 \pm 39.3$ \\
CG = control group; IG = intervention group; BMI = body mass index; & \\
SBP = systolic blood pressure; DBP = diastolic blood pressure. & \\
${ }^{* A l l}$ values are expressed as means \pm standard deviations. & \\
&
\end{tabular}

further noted that none of the participants in the CG had accumulated any aerobic steps at baseline.

\section{Questionnaire evaluation on perceptions of the intervention}

Most participants expressed that the pedometer intervention was 'catchy' and that the pedometer served as a useful motivational aid and a reminder of the need to increase steps per day. Participants also found the individualised feedback to be valuable in summarising their ambulatory PA over the 2-week period, and became more aware of the need to increase their steps per day.

Barriers identified by participants related mainly to adherence to the intervention, largely due to having a sedentary occupation and experiencing time limitations to incorporating PA into daily routine. Participants also found it very difficult to achieve or increase their aerobic steps, despite an overall increase in the total volume of steps per day. Participants indicated that their interest in the intervention decreased as the intervention continued. Despite this, participants also felt that a longer intervention might have assisted them in further increasing their steps per day.

The difference between baseline and follow-up measures within the study group was determined in order to establish the potential effect of the intervention on clinical measures (Table 2).

An interesting observation was that the average number of aerobic steps per day decreased at follow-up in the IG, notwithstanding the small number of steps and the very large standard deviation. The time spent accumulating aerobic steps increased in the IG. The daily steps, however, increased by almost 1000 steps in the IG v. 97 steps in the CG. No aerobic steps were accumulated at baseline and follow-up in the CG for comparative purposes.

\section{Power calculation and sample size estimation for a future RCT}

A power analysis for a two-group, independent sample $t$-test was conducted using the GPower data analysis website. ${ }^{[17]}$ Based on a minimum improvement of 1000 steps/day, as established from this 
Table 2. Net change in characteristics at follow-up $(N=19)$

\begin{tabular}{|c|c|c|}
\hline Variable ${ }^{*}$ & CG $(N=8)^{\dagger}$ & IG $(N=11)^{\dagger}$ \\
\hline Waist circumference (cm) & $-0.38 \pm 2.0$ & $+0.73 \pm 2.7$ \\
\hline Body fat (\%) & $+0.26 \pm 1.3$ & $+1.34 \pm 1.9$ \\
\hline BMI $\left(\mathrm{kg} / \mathrm{m}^{2}\right)$ & $+0.13 \pm 0.5$ & $+0.26 \pm 0.58$ \\
\hline Body weight (kg) & $+0.3 \pm 1.3$ & $+0.67 \pm 1.6$ \\
\hline SBP (mmHg) & $-0.8 \pm 8.0$ & $+0.3 \pm 8.9$ \\
\hline DBP (mmHg) & $-3.1 \pm 3.7$ & $-2.5 \pm 7.6$ \\
\hline Daily steps (steps/day) & $+97 \pm 750$ & $+996 \pm 1748$ \\
\hline Daily aerobic steps (steps/day) & 0 & $-54 \pm 2746$ \\
\hline Daily aerobic time (min) & 0 & $+0.9 \pm 23.0$ \\
\hline \multicolumn{3}{|c|}{$\begin{array}{l}C G=\text { control group; } I G=\text { intervention group; } B M I=\text { body mass index; } \\
S B P=\text { systolic blood pressure; } D B P=\text { diastolic blood pressure. }\end{array}$} \\
\hline \multicolumn{3}{|c|}{ *All values are expressed as means \pm standard deviations. } \\
\hline $\begin{array}{l}\text { A + symbol indicates an improvement in the } \\
\text { in the desired direction. }\end{array}$ & esired direction; a & ol indicates a decreas \\
\hline
\end{tabular}

pilot study, a sample size of approximately 85 participants per arm of the study is required to ensure $80 \%$ statistical power and a $p$-value $<0.05$. Considering this possibility and the likelihood of performing subgroup analyses based on factors such as age and gender, as well as the possibility of loss to follow-up, we estimate that a sample size of 150 participants in the IG and CG, respectively, would be an appropriate target sample.

\section{Discussion}

The purpose of this study was to evaluate the feasibility of a pilot intervention aimed at improving the daily ambulatory PA in an employed, South African adult population. A further objective was to use the outcomes of the study (i.e. changes in steps per day between baseline and follow-up) to inform the development of, and calculate the recommended sample size for, a pedometer-based intervention protocol, to be administered as a future RCT.

\section{Evaluation on perceptions of the intervention}

Adherence to PA emerged as a limitation to increasing steps per day. This was expressed to be largely due to being sedentary at work and experiencing time limitations in incorporating PA into daily routine.

Several studies, as reported by Fox et al. ${ }^{[18]}$ show similar findings and a growing body of research supports the need to build PA into daily routine. ${ }^{[9]}$ Interventions may also need to focus on institutionallevel efforts to support building activity into daily routines. ${ }^{[18]}$ Such support measures can include creating walk-paths around the work environment or incorporating a PA programme into the work day. ${ }^{[18]}$ Additional measures include using motivational prompts encouraging/ reminding people to be more active during work-time, or foot-markers directing people to the stairwell instead of the lifts. ${ }^{[18]}$

\section{Pedometer outcomes}

Due to the small sample size, it would be inappropriate to draw conclusions based on statistical analysis from the pedometer results. A useful finding, nevertheless, was the overall increase in total steps per day in the IG of approximately 1000 steps/day.
Recently published papers and systematic reviews of pedometer-based interventions conducted between 1966 and $2007^{[11,18,19]}$ show an average improvement of 1500 - 3000 steps/day. These studies, however, generally showed a higher baseline steps per day volume than our study group (7 500 steps/day v. 5000 steps/day, respectively). No reference has been made to intensity-based steps in any of these previous interventions. Our study, therefore, provides some information on intensity of steps per day and identifies intensity-based messages as a strategy towards improved health outcomes.

\section{Modifications of the pilot study for future RCT application}

The allocation of participants to a future trial should be based on a theoretical model for behavioural change; e.g. the intervention should be targeted towards participants at a specific stage of behavioural change, so as to be mentored similarly to ensure improved uptake, compliance and overall impact of the intervention in terms of increased PA. As such, the intervention can be targeted specifically at individuals not meeting current guidelines and in the contemplation stage of behavioural change, as per the trans-theoretical model of behavioural change. ${ }^{[20]}$

The emailed messages delivered to participants should include additional information and not only a summation of steps accumulated. Typically, the information must also indicate how the results relate to current PA guidelines or intensity-based steps per day targets. This will contribute towards the reinforcement of public health recommendations that may prompt individuals to self-reflect on current PA levels, in keeping with pre-determined goals.

The importance of intensity-based steps should be reinforced further during the bi-weekly feedback emails, with particular emphasis on current PA guidelines. This includes a combination of duration and intensity-based steps data rather than volume-based information. This may require revision of the current 'aerobic' classification used by the pedometer, as determined in recent studies. ${ }^{[8,21-25]}$

The general information provided to the IG and CG was the same irrespective of individual progress towards improved ambulatory PA. Depending on the extent of available resources, tailored feedback can be provided at an individual level based on the level of progress. In a similar manner, barriers towards progress can be identified with improvement strategies provided on a more individual basis.

\section{Study strengths and limitations}

The study is among the first pedometer-based interventions conducted in South Africa. More importantly, this pilot intervention informs the development and protocol of a pedometer-based intervention that can be applied on a larger scale and in a wider context. In terms of study limitations, the small sample size and the element of selection bias (as the study involved selection from a convenience sample of persons) were primary limitations. Also, the sample was taken from a health sciences faculty, and might not have been representative of staff in other faculties in terms of knowledge and interest in PA and health.

Waist circumference was measured using a tape measure around the skin of the waist. More recent recommendations ${ }^{[26]}$ for measuring waist circumference can be applied in future studies.

We noted a difference in BMI between the IG and CG at baseline and acknowledge that this might have had an effect on the steps per day data recorded at follow-up. Similarly, none of the participants 
in the CG accumulated any aerobic steps at baseline. As such, the difference between the two groups at baseline might have contributed to the difference observed between the groups at follow-up.

The CG received the same general motivational messages as the IG bi-weekly, which might have led to increased PA in the CG.

No theoretical model for behavioural change was applied; this implied that, using a convenience sample approach, participants were at various stages of change towards improving ambulatory PA. The study might therefore have included participants who were already meeting current PA guidelines and were interested in further improving this through the intervention. The emailed feedback messages provided to IG participants only provided a summation of pedometer results, but did not indicate how this related to current guidelines or intensity-based steps per day targets as a method of reinforcement.

\section{Conclusion}

This pilot study provides useful information on the potential for PA improvements through pedometry in an employed, adult group. In so doing, the study provides a basis to further pedometer-based interventions that can be applied in other contexts and settings and on a larger scale. Consequently, this pilot intervention informs the development and protocol of a large-scale intervention to be applied as an RCT.

Funding acknowledgements. Durban University of Technology (DUT) and the National Research Foundation (Thuthuka).

\section{References}

1. Haskell WL, I-Min L, Pate RR, et al. Physical activity and public health: Updated recommendation for adults from the American College of Sports Medicine and the American Heart Association. Circulation 2007;116(9):1081-1093. [http://dx.doi. org/10.1161/CIRCULATIONAHA.107.185649]

2. Tudor-Locke C. Promoting lifestyle physical activity: Experiences with the First Step Program. Am J Lifestyle Med 2009;3(1 suppl):50S-54S

3. Pillay JD, Kolbe-Alexander TL, Achmat M, Carstens M, Lambert EV. Are pointof-decision prompts in sports science and medicine centre effective in changing the prevalence of stair usage? A preliminary study. South African Journal of Sports Medicine 2009;21(2):58-64.

4. Tudor-Locke C. Taking Steps Toward Increased Physical Activity: Using Pedometers To Measure And Motivate. Washington DC, USA: President's Council on Physical Fitness and Sports, 2002.

5. Richardson CR, Newton TL, Abraham JJ, Sen A, Jimbo M, Swartz AM. A metaanalysis of pedometer-based walking interventions and weight loss. Ann Fam Med 2008;6(1):69-77. [http://dx.doi.org/10.1370/afm.761]
6. Freedson PS, Miller K. Objective monitoring of physical activity using motion sensors and heart rate. Res Q Exerc Sport 2000;71(2 suppl):S21-S29.

7. Welk GJ, Corbin CB, Dale D. Measurement issues in the assessment of physical activity in children. Res Q Exerc Sport 2000;71(2 suppl):59-73.

8. Tudor-Locke C, Sisson SB, Collova T, Lee SM, Swan PD. Pedometer-determined step count guidelines for classifying walking intensity in a young ostensibly healthy population. Can J Appl Physiol 2005;30(6):666-676.

9. Bravata DM, Smith-Spangler CS, Sundaram V, et al. Using pedometers to increase physical activity and improve health - a systematic review. JAMA 2007;298(19):22962304 .

10. Harrington NG, Noar SM. Reporting standards for studies of tailored interventions (R2). Health Educ Res 2012;27(2):331-342. [http://dx.doi.org/10.1093/her/cyr108]

11. Ogilvie D, Foster CE, Rothnie H, et al. Interventions to promote walking: Systematic review. BMJ 2007;334:1204-1214.

12. Lenfant C. Shattuck lecture-clinical research to clinical practice-lost in translation? $\mathrm{N}$ Engl J Med 2003;349(9):868-874.

13. Glasgow RE, Emmons KM. How can we increase translation of research into practice? Types of evidence needed. Annu Rev Public Health 2007;28(1):413-433.

14. Chisholm DM, Collis ML, Kulak LL, Davenport W, Gruber N, Stewart GW. PAR-Q Validation Report: The Evaluation of a Self-administered Pre-exercise Screening Questionnaire for Adults. Victoria, Canada: BC Ministry of Health and Welfare, 1978

15. Bosy-Westphal A, Later W, Hitze B, et al. Accuracy of bioelectrical impedence consumer devices for measurement of body composition in comparison to whole body magnetic resonance imaging and dual x-ray absorptiometry. Obes Facts 2008;1:319-324. [http://dx.doi.org/10.1159/000176061]

16. Incorporated OH. Instruction manual: Pocket pedometer-model HJ-720ITC2007.

17. $\mathrm{G}^{\star}$ Power version 3.0.10. Power analysis for two-group independent sample $t$-test. http://www.ats.ucla.edu/stat/gpower/indepsamps.htm (accessed 16 October 2012).

18. Fox AM, Mann DM, Ramos MA, Kleinman LC, Horowitz CR. Barriers to physical activity in East Harlem, New York. J Obes 2012;719140. [http://dx.doi. org/10.1155/2012/719140]

19. Chan CB, Ryan DAJ, Tudor-Locke C. Health benefits of a pedometer-based physical activity intervention in sedentary workers. Prev Med 2004;39:1215-1222.

20. Prochaska JO, Velicer WF. The transtheoretical model of health behavior change. Am J Health Promot 1997;12(1):38-48.

21. Marshall SJ, Levy SS, Tudor-Locke CE, et al. Translating physical activity recommendations into a pedometer-based step goal: 3000 steps in 30 minutes. Am J Prev Med 2009;36(5):410-415. [http://dx.doi.org/10.1016/j.amepre.2009.01.021]

22. Pillay JD, Kolbe-Alexander TL, van Mechelen W, Lambert EV. Steps that count - the association between the number and intensity of steps accumulated and fitness and health measures. J Phys Act Health 2012 (in press). [http://dx.doi.org/10.1186/14712458-12-880]

23. Pillay JD, Kolbe-Alexander TL, Proper KI, van Mechelen W, Lambert EV. Steps that count - physical activity recommendations, brisk walking and steps per minute - how do they relate? J Phys Act Health 2013 (in press).

24. Abel M, Hannon J, Mullineaux D, Beighle A. Determination of step rate thresholds corresponding to physical activity intensity classifications in adults. J Phys Act Health 2011;8(1):45-51.

25. Tudor-Locke C, Camhi SM, Leonardi C, et al. Patterns of adult stepping in the 2005/2006 NHANES. Prev Med 2011;53(3):178-181. [http://dx.doi.org/10.1016/j. ypmed.2011.06.004]

26. Stewart A, Marfell-Jones M, Olds T, de Ridder H. International Standards for Anthropometric Assessment. Lower Hutt, New Zealand: ISAK, 2011. 